\title{
Energetics of quantum correlations
}

\author{
Raoul Dillenschneider and Eric Lutz \\ Department of Physics, University of Augsburg, D-86135 Augsburg, Germany \\ PACS 03.67.-a - Quantum information \\ PACS 05.70.-a - Thermodynamics \\ PACS 03.65.Yz - Decoherence; open systems; quantum statistical methods
}

\begin{abstract}
We consider a photo-Carnot engine that consists of a single-mode radiation field in an optical cavity. One the heat reservoirs is made of a beam of thermally entangled pairs of two-level atoms that interact resonantly with the cavity. We express the thermodynamic efficiency of the engine in terms of the quantum discord of the atomic pair and find that it can exceed its classical value. Our results show that useful work can be extracted from quantum correlations, indicating that the latter are a valuable resource in quantum thermodynamics.
\end{abstract}

Introduction. - Nonclassical correlations are one of the most intriguing consequences of quantum theory. A major discovery of the last decade is that the efficiency of , some computing and information processing tasks can be greatly enhanced when using quantum correlated states [1]. Quantum correlations are traditionally associated ' with entanglement, that is, nonseparability. However, it has recently been realized that the notion of nonclassical correlations is more general [2-4]. An informationtheoretic measure of the quantumness of correlations, the quantum discord, has been introduced by Ollivier and Zurek [3]. It is defined as the difference of two expres' sions of the mutual information that are classically identical (see Eq. 11] below). The quantum discord can be nonzero for states that are separable, indicating the presence , of quantum correlations in nonentangled states. Remarkably, a recent experiment has demonstrated that states , with non-zero discord can lead to an exponential speedup ' in a model of quantum computation (deterministic quantum computation with one quantum bit - DQC1), in the absence of entanglement $[5,6]$.

A thermodynamic approach to quantify quantum correlations has been developed in Refs. [7-10], exploiting the intimate connection existing between thermodynamics and information theory. The basic quantity in this context is the work deficit, defined as the difference of thermodynamic work that can be extracted globally and locally from a heat bath using a correlated bipartite state. The work deficit has been shown to be equal to the quantum discord when one-way communication is allowed $[8,9]$. In this paper, we consider the problem of gaining useful work from quantum correlated states. Specifically, we discuss a scheme that permits extraction of thermodynamical work from an ensemble of thermal quantum correlated qubits with non-zero discord.

We consider the photo-Carnot engine introduced by Scully and coworkers [11]. The latter consists of a single mode of a quantized radiation field inside a resonant high-quality optical cavity. One of the mirrors of the cavity (the piston) is driven by the radiation pressure of the thermal photons of the field, while the other mirror is used to exchange heat with an external reservoir. The coupling to a second (quantum) reservoir is achieved by sending individual thermal atoms through the cavity and letting them interact with the radiation field. Two cases can then be distinguished [11]: When regular two-level atoms are sent through the cavity, the maximum efficiency of the quantum engine after a Carnot cycle is found to be given by the classical efficiency, $\eta_{C}=1-T_{c} / T_{h}$, where $T_{c}$ and $T_{h}$ are the respective temperatures of the cold and hot reservoir (which here corresponds to the atomic reservoir). The situation changes dramatically when threelevel atoms are considered instead. By preparing the two (quasi-degenerate) ground states in a coherent superposition and by properly tuning the relative phase between the two states, the temperature of the cavity can be effectively increased. As a consequence, the quantum efficiency can become larger than the classical one, $\eta>\eta_{C}$. Thermodynamic efficiency can therefore be improved by using quantum coherence as a resource.

In order to investigate the effect of quantum correlations in the quantum Carnot engine described above, we 
consider pairwise correlated two-level atoms in a thermal entangled state [12]. The notable feature of these states is the existence of entanglement in thermal equilibrium at finite temperature. Thermal entanglement has been extensively studied in solid-state systems, such as various Ising or Heisenberg spin models [13-15]. Signatures of thermal entanglement in macroscopic systems have in addition been observed in recent experiments [16-18].

In the following, we calculate the thermodynamic efficiency of the photo-Carnot engine when a beam of twolevel atoms in thermal entangled states is sent through the optical cavity. In the limit of very large number of atoms, the beam can be considered as a heat reservoir and the efficiency of the quantum engine reduces to the known classical value when atoms are not correlated. We treat two different cases: In the first case, the two atoms of a correlated pair fly sequentially through the cavity, whereas in the second case, only one of the atoms interacts with the radiation field. In both situations, we find that the presence of correlations modifies detailed-balance between absorption and emission of photons in the cavity and changes it effective temperature; the temperature is lowered in the two-atom case, while it is raised in the one-atom case. As a result the quantum Carnot engine can outperform the classical engine, showing that quantum correlations are a valuable resource.

Thermal entanglement. - Let us consider two identical two-level atoms with frequency $\omega$ coupled via a XYHeisenberg interaction of the form,

$$
H=\hbar \omega S_{1}^{z}+\hbar \omega S_{2}^{z}+\hbar \lambda\left(S_{1}^{+} S_{2}^{-}+S_{1}^{-} S_{2}^{+}\right)
$$

where $\lambda$ is the controllable strength of the interaction. We have here defined the spin operators $S_{j}^{z}=\sigma_{j}^{z} / 2=$ $\left(|g\rangle\left\langle\left. g\right|_{j}-\mid e\right\rangle\left\langle\left. e\right|_{j}\right) / 2\right.$ and $S_{j}^{ \pm}=\left(S_{j}^{x} \pm i S_{j}^{y}\right)$, where $\sigma^{x, y, z}$ are the usual Pauli operators. Ground and excited states are denoted by $|g\rangle$ and $|e\rangle$. In the context of cavity QED, a scheme to entangle in a controlled manner two identical atoms via the XY-Heisenberg interaction (1) has been recently proposed in Ref. [19] and implemented experimentally [20]. The state of the two atoms in thermal equilibrium at temperature $T$ is described by the density operator, $\rho=Z^{-1} \exp (-\beta H)$, where $Z=\operatorname{Tr} \exp (-\beta H)=$ $2(\cosh (\beta \hbar \omega)+\cosh (\beta \hbar \lambda))$ is the partition function and $\beta=(k T)^{-1}$. In the natural basis $\{|g g\rangle,|g e\rangle,|e g\rangle,|e e\rangle\}$, the thermal density matrix can be written as,

$$
\begin{aligned}
\rho= & \frac{1}{Z}\left(e^{\beta \hbar \omega}|g g\rangle\left\langle g g\left|+e^{-\beta \hbar \omega}\right| e e\right\rangle\langle e e|\right. \\
& \left.+e^{\beta \hbar \lambda}\left|\Psi_{-}\right\rangle\left\langle\Psi_{-}\left|+e^{-\beta \hbar \lambda}\right| \Psi_{+}\right\rangle\left\langle\Psi_{+}\right|\right),
\end{aligned}
$$

where we have introduced the maximally entangled Bell states, $\left|\Psi_{ \pm}\right\rangle=( \pm|g e\rangle+|e g\rangle) / \sqrt{2}$, which, together with

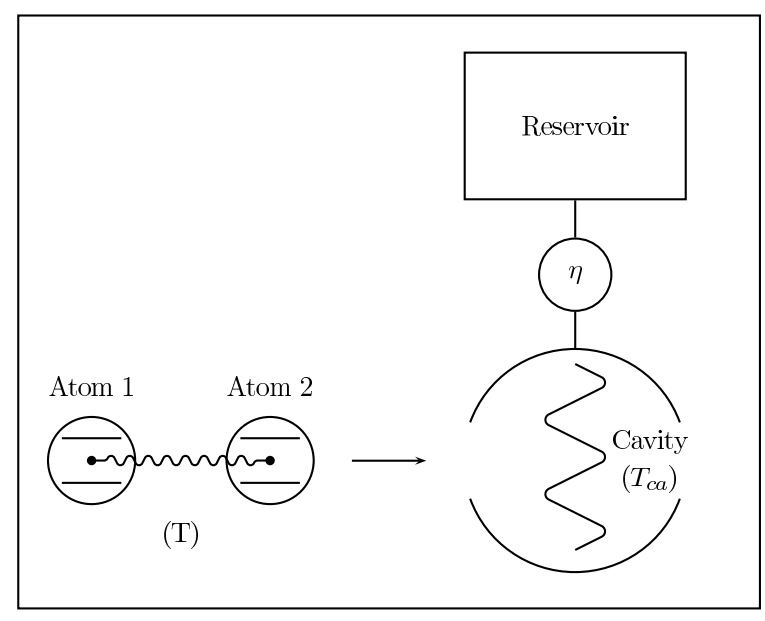

Fig. 1: Schematic representation of the photo-Carnot engine: A beam of quantum correlated two-level atoms (quantum reservoir at temperature $T$ ) is sent through a resonant optical cavity (system at temperature $T_{c a}$ ). Work is produced by putting the cavity cyclically in contact with another reservoir at a different temperature, with a quantum efficiency $\eta$.

the states $|g g\rangle$ and $|e e\rangle$, form an orthogonal basis. It is worth noticing that the interaction parameter $\lambda$ controls the weight of the two Bell states inside the mixture. For further reference, we also define the following matrix elements of $\rho$ : the matrix elements in the ground and excited states, $\rho_{g}=\langle g g|\rho| g g\rangle=Z^{-1} \exp (\beta \hbar \omega)$ and $\rho_{e}=\langle e e|\rho| e e\rangle=Z^{-1} \exp (-\beta \hbar \omega)$, as well as the diagonal, $\rho_{d}=\langle g e|\rho| g e\rangle=\langle e g|\rho| e g\rangle=Z^{-1} \cosh (\beta \hbar \lambda)$ and nondiagonal elements, $\rho_{n d}=\langle g e|\rho| e g\rangle=\langle e g|\rho| g e\rangle=$ $-Z^{-1} \sinh (\beta \hbar \lambda)$. The nondiagonal matrix elements vanish when $\lambda=0$.

The entanglement properties of the thermal state (2) have been investigated in Ref. [21]. The concurrence can be evaluated in closed form and reads

$$
\mathcal{C}=\max \left\{0, \frac{\sinh (\beta \hbar \lambda)-1}{\cosh (\beta \hbar \omega)+\cosh (\beta \hbar \lambda)}\right\} .
$$

For $\beta \hbar \lambda>\operatorname{arcsinh}(1) \simeq 0.88$, the concurrence is nonzero and the two atoms described by Eq. (2) are therefore thermally entangled. However, for $\beta \hbar \lambda \leq \operatorname{arcsinh}(1)$, the concurrence vanishes and the mixture is separable. The thermal state (2) is thus entangled for sufficiently low temperature and/or strong coupling $\lambda$.

Two atoms through cavity. - We now focus on the situation in which atoms of thermal entangled pairs pass through the cavity at random time intervals, as depicted in Fig 1, Each atom, $j=1,2$, interacts with the single mode of the radiation field via a resonant JaynesCummings coupling, $H_{j}^{i n t}=-g \hbar\left(a S_{j}^{+}+a^{\dagger} S_{j}^{-}\right)$, with coupling constant $g$. The Hamiltonian of the cavity mode is $H_{c a}=\hbar \omega a^{\dagger} a$, where $a$ and $a^{\dagger}$ are the ladder operators of the oscillator. We are interested in the stationary prop- 
erties of the single cavity mode described by the operator $\rho_{c a}(t)$, when a large number of similarly prepared pairs of atoms is sent through the cavity. The master equation obeyed by $\rho_{c a}(t)$ can be derived in the limit of weak coupling by using standard techniques [22] and reads,

$$
\begin{aligned}
& \frac{\partial \rho_{c a}}{\partial t}=i \omega\left[\rho_{c a}, a^{\dagger} a\right] \\
& -\frac{r_{1}}{2} \phi^{2}\left(a a^{\dagger} \rho_{c a}-2 a^{\dagger} \rho_{c a} a+\rho_{c a} a a^{\dagger}\right) \\
& -\frac{r_{2}}{2} \phi^{2}\left(a^{\dagger} a \rho_{c a}-2 a \rho_{c a} a^{\dagger}+\rho_{c a} a^{\dagger} a\right),
\end{aligned}
$$

where $\phi=g \tau$ with $\tau$ the time spent by atoms inside the cavity. The coefficients $r_{1}$ and $r_{2}$ are the arrival rates for atoms being respectively in the excited and ground state. They are related to the probabilities of emission and absorption of a photon in the cavity and are explicitly given by $r_{1}=\left(\rho_{e}+\rho_{d}+\rho_{n d}\right)$ and $r_{2}=\left(\rho_{g}+\rho_{d}+\rho_{n d}\right)$. The asymptotic steady state solution of the master equation (44) is the thermal distribution,

$$
\rho_{c a}^{s}=\left(1-e^{-\beta_{c a} \hbar \omega}\right) e^{-\beta_{c a} \hbar \omega a^{\dagger} a} .
$$

The inverse temperature of the cavity, $\beta_{c a}=\left(k T_{c a}\right)^{-1}$, is determined by the coefficients $r_{1}$ and $r_{2}$ through the detailed-balance condition, $\exp \left(-\beta_{c a} \omega\right)=r_{1} / r_{2}$. When the interaction parameter $\lambda$ is zero, the temperature of the cavity is equal to the atomic temperature, $T_{c a}=T$. On the other hand, when $\lambda \neq 0$, the diagonal and nondiagonal matrix elements, $\rho_{d}$ and $\rho_{n d}$, modify detailed-balance between emission and absorption. As a result, the temperature of the cavity is effectively changed. We will see below that $\lambda \neq 0$ indicates the presence of quantum correlations between two paired atoms.

The temperature of the cavity can be readily expressed in terms of the frequency $\omega$ of the two-level atoms and the interaction parameter $\lambda$. We have,

$$
\frac{\beta_{c a}}{\beta}=1-\frac{1}{\beta \hbar \omega} \ln \left(\frac{1+e^{\beta \hbar \omega} e^{-\beta \hbar \lambda}}{e^{\beta \hbar \omega}+e^{-\beta \hbar \lambda}}\right) .
$$

The ratio $\beta_{c a} / \beta$ is always larger or equal than one, implying that the temperature of the cavity is smaller than the temperature of the reservoir when $\lambda \neq 0$.

One atom through cavity. - We next consider the case where only one atom of the thermal entangled pair is sent through the cavity. The density operator of that atom is obtained from the total density matrix (2) by tracing over the second atom. Due to the symmetry of the mixture, the two reduced operators are identical. By repeating the previous analysis, we find that the stationary state of the cavity field is thermally distributed with an inverse temperature, $\beta_{c a}^{\prime}=\left(k T_{c a}^{\prime}\right)^{-1}$, satisfying $\exp \left(-\beta_{c a}^{\prime} \omega\right)=r_{1}^{\prime} / r_{2}^{\prime}$, with $r_{1}^{\prime}=\rho_{e}+\rho_{d}$ and $r_{2}^{\prime}=\rho_{g}+\rho_{d}$. Here, the coefficients $r_{1}^{\prime}$ and $r_{2}^{\prime}$ depend on the interaction parameter $\lambda$ only through the diagonal matrix elements $\rho_{d}$, since the nondiagonal elements do not appear. For the inverse temperature of the cavity, we obtain,

$$
\frac{\beta_{c a}^{\prime}}{\beta}=1-\frac{1}{\beta \hbar \omega} \ln \frac{1+e^{\beta \hbar \omega} \cosh (\beta \hbar \lambda)}{e^{\beta \hbar \omega}+\cosh (\beta \hbar \lambda)} .
$$

Contrary to the two-atom case, we observe that the inverse temperature ratio $\beta_{c a}^{\prime} / \beta$ is smaller or equal than one, indicating that the temperature of the cavity is larger than $T$ when $\lambda \neq 0$.

Absorption and emission. - Deeper insight into the previous results can be gained by directly examining the probabilities of absorption and emission of a photon by the cavity mode. The latter can be easily calculated from the master equation (2) by evaluating the transition rates per unit time, $t_{ \pm}$, from an oscillator state $|n\rangle$ to the adjacent states $|n \pm 1\rangle$ [23]. They are given by

$$
\begin{array}{lll}
t_{+}=r_{1} \phi^{2}(n+1), & & \text { (absorption) } \\
t_{-}=r_{2} \phi^{2} n, & & \text { (emission) }
\end{array}
$$

and similar expressions for the transition rates, $t_{ \pm}^{\prime}$, in the one-atom case. The dependence of the different rates on the interaction parameter $\lambda$ is shown in Fig. 2, When a beam of correlated atomic pairs is sent through the cavity, a larger value of $\lambda$ leads to a stronger reduction of the absorption rate than the emission rate. As a consequence, less energy is deposited in the cavity and its temperature is therefore effectively lowered. On the other hand, when only one atom of the pair passes through the cavity, the absorption probability is enhanced, while emission is suppressed. In contrast to the previous situation, more energy is thus provided to the cavity. This results in an effective temperature rise.

The above results seem to contradict the zeroth law of thermodynamics which states that two objects in thermal equilibrium have the same temperature; one would then expect the temperature of the system to be equal to the temperature of the reservoir [24]. The key observation to resolve this apparent paradox is that, contrary to the usual situation in macroscopic thermodynamics, here the system (cavity mode) does not interact with the reservoir (atomic beam) as a whole, but sequentially with its individual parts (atoms). Let us consider the total density operator of a pair of atoms as given by Eq. (2). The latter corresponds to the equilibrium Gibbs state $\rho=Z^{-1} \exp (-\beta H)$. In the absence of correlations, the total density operator of the pair is the direct product, $\rho=\rho_{\mathcal{A}_{1}} \otimes \rho_{\mathcal{A}_{2}}$, of the reduced density operators of the individual atoms, $\rho_{\mathcal{A}_{i}}=\operatorname{Tr}_{\mathcal{A}_{j}} \rho$; they are also given by Gibbs states, $\rho_{\mathcal{A}_{i}}=Z_{i}^{-1} \exp \left(-\beta H_{i}\right)$, since $\lambda=0$. In the presence of correlations, however, the total state does not factorize and the density operators of atoms $\mathcal{A}_{1}$ and $\mathcal{A}_{2}$ are no longer of the Gibbs form. Therefore, although the 


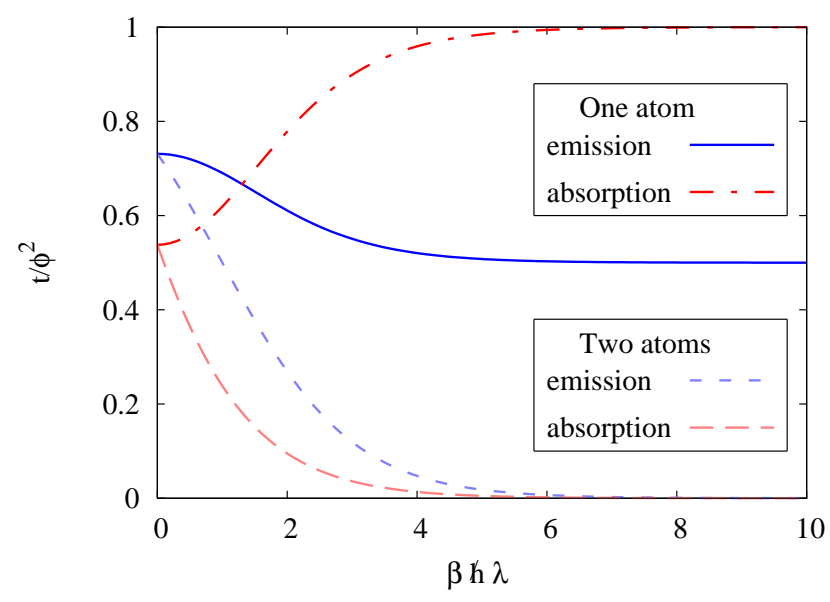

Fig. 2: Probabilities of absorption and emission, $t_{ \pm}$, of a photon by the cavity mode, Eqs. (8) and (9), from the oscillator state $n=1$, as a function of the interaction parameter $\beta \hbar \lambda$ for a fixed value of the temperature $\beta \hbar \omega=1$.

pair as a whole is in an equilibrium state, the individual atoms of the pair, with which the cavity mode interacts, are not. The reduced density operators of the atoms are explicitly given by,

$$
\rho_{\mathcal{A}_{1}}=\rho_{\mathcal{A}_{2}}=r_{2}|g\rangle\left\langle g\left|+r_{1}\right| e\right\rangle\langle e| .
$$

The level occupancies of ground and excited states take their equilibrium values, $e^{\beta \hbar \omega} / 2 \cos (\beta \hbar \omega)$ and $e^{-\beta \hbar \omega} / 2 \cos (\beta \hbar \omega)$, only when $\lambda=0$. The resonant interaction of the cavity mode with the nonequilibium atoms then leads to the thermal state, Eq. (5), with a temperature different from the reservoir temperature. This unusual feature is a consequence of the microscopic size of the photo-Carnot engine; it occurs for the quantum correlated as well as for the quantum coherent atomic reservoir [11]. It is important to emphasize that the steady-state density operator of the cavity mode, Eq. (5), is always given by a thermal state with a well-defined temperature for all values of the interaction parameter $\lambda$.

Interestingly, the temperature of the cavity differs from the atomic temperature in both cases, even when $\beta \hbar \lambda \leq$ $\operatorname{arcsinh}(1)$, that is, even when the two-atom state is separable. This result shows that entanglement by itself is not responsible for the observed deviations. In the following, we express the modified cavity temperature in terms of the quantum discord.

Quantum discord. - The quantum discord is defined as the difference between two expressions of the mutual information that are classically equal [3],

$$
\delta(1 \mid 2)=\mathcal{I}(1: 2)-\mathcal{J}(1: 2) .
$$

The quantum mutual information $\mathcal{I}(1: 2)$ is given by $\mathcal{I}(1$ : $2)=H(1)+H(2)-H(1,2)$. Here $H(j)=-\operatorname{Tr} \rho_{j} \log _{2} \rho_{j}$, is the von Neumann entropy of the reduced density operator of atom $j$, and $H(1,2)=-\operatorname{Tr} \rho \log _{2} \rho$ is the joint entropy of the total density matrix (2). The alternative quantum generalization of the mutual information is $\mathcal{J}(1: 2)=H(1)-H\left(1 \mid\left\{\Pi_{2, l}\right\}\right)$; it describes the information gained about atom 1 as a result of a set of measurement $\left.\left\{\Pi_{2, l}\right\}\right)$ on atom 2 . The conditional entropy, $H\left(1 \mid\left\{\Pi_{2, l}\right\}\right)=\sum_{l} p_{l} H\left(1 \mid \Pi_{2, l}\right)$, is the sum of the von Neumann entropies $H\left(1 \mid \Pi_{2, l}\right)$ of the conditional density operators, $\rho_{1 \mid \Pi_{2, l}}=\Pi_{2, l} \rho \Pi_{2, l} / p_{l}$, after a perfect measurement of atom 2. The operators $\left\{\Pi_{2, l}\right\}$ define a set of orthogonal projectors on atom 2 and $p_{l}=\operatorname{Tr}_{1,2} \Pi_{2, l} \rho$ denotes the probability of occurrence of state $\rho_{1 \mid \Pi_{2, l}}$ in a given measurement. The mutual information $\mathcal{J}(1: 2)$ usually depends on the measurement basis and the discord has to be minimized over all sets $\left\{\Pi_{2 l}\right\}$. Using the parametrized basis, $\{\cos \theta|g\rangle-\sin \theta|e\rangle,-\sin \theta|g\rangle-\cos \theta|e\rangle\}$, we find the minimum of the discord for $\theta=\pi / 4$ :

$$
\begin{array}{r}
\delta(1 \mid 2)=-\frac{1}{\ln (2)}\left[2(\beta \hbar \lambda) \rho_{n d}+\sum_{\alpha=g, e} \rho_{\alpha} \ln \left(\frac{\rho_{\alpha}+\rho_{d}}{\rho_{\alpha}}\right)\right. \\
\left.+\rho_{d} \ln \left(Z^{2}\left(\rho_{g}+\rho_{d}\right)\left(\rho_{e}+\rho_{d}\right)\right)+\sum_{\varepsilon= \pm} \Phi_{\varepsilon} \ln \Phi_{\varepsilon}\right]
\end{array}
$$

where we have defined $\Phi_{\varepsilon}=\left(1+\varepsilon \sqrt{\left(\rho_{e}-\rho_{g}\right)^{2}+4 \rho_{n d}^{2}}\right) / 2$. The discord vanishes when the interaction parameter $\lambda$ is zero, corresponding to the absence of quantum correlations between the two atoms. For maximally correlated states, the discord is unity and is, in this particular case, equal to the concurrence $\mathcal{C}$. In general, however, the discord is different from the concurrence and can even be nonzero when the state is separable.

Figure 3 shows the ratio of the temperature of the cavity and the reservoir temperature, Eqs. (6) and (7), as a function of the quantum discord (12) for a fixed value of $\beta \hbar \omega$. We first observe that the temperature of the cavity is equal to the reservoir temperature in the absence of quantum correlations when the discord is zero. We further clearly recognize that the temperature of the cavity increases with the discord in the case where only one of the two correlated atoms passes through the cavity, and decreases when both atoms fly through the cavity. We can therefore conclude that the deviation of the cavity temperature from the reservoir temperature is induced by the quantum correlations of the thermal entangled pair. The maximum temperature deviation is obtained for maximal quantum correlations, $\delta(1 \mid 2)=1$.

For general correlated states, quantum and classical correlations coexist and quantifying them is a nontrivial task. Following Henderson and Vedral [25], we compute classical correlations in a quantum system as the maximum over all measurements of the quantum mutual information $\mathcal{J}(1: 2)$, 


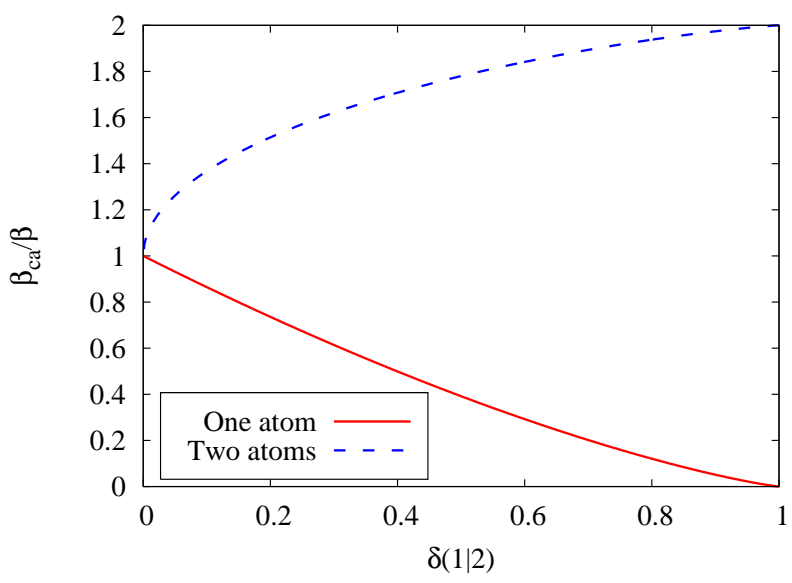

Fig. 3: Relative inverse temperature of the cavity, $\beta_{c a} / \beta$, Eqs. (6) and (7), as a function of the quantum discord $\delta(1 \mid 2)$, Eq. (12), for the parameter $\beta \hbar \omega=10^{-3}$. The full (dashed) line corresponds to the situation in which one (two) atom(s) of the thermal entangled pair goes through the optical cavity.

$$
C(1 \mid 2)=\max _{\left\{\Pi_{2, l}^{\prime}\right\}} \mathcal{J}(1: 2) .
$$

For the bipartite thermal entangled state, Eq. (5), we find that the set of measurements that minimizes the quantum discord also maximizes classical correlations. In view of the definition of these two quantities, we thus obtain that $\mathcal{I}(1: 2)=\delta(1 \mid 2)+C(1 \mid 2)$. In other words, classical and quantum correlations do add up in this case to the total correlations, as given by the mutual information. We have verified that this result also holds for generalized POVM measurements [26].

The inverse temperature dependence of the four correlation measures, $\mathcal{I}(1: 2), \delta(1 \mid 2), C(1 \mid 2)$ and $\mathcal{C}$, is plotted in Fig团 for a fixed value of the interaction parameter $\lambda<\omega$. In the limit of high temperatures, $\beta \hbar \omega \ll 1$, the atomic pair is separable $(\mathcal{C}=0)$ and quantum discord is equal to classical correlations, $\delta(1 \mid 2) \simeq C(1 \mid 2)$. It should be stressed that classical correlations are in general much smaller than quantum correlations. For small interaction parameter, $\beta \hbar \lambda \ll 1$, Eqs. (12) and (13) simplify to $\delta(1 \mid 2) \simeq C(1 \mid 2) \simeq(\beta \hbar \lambda)^{2} / 8 \ln 2$. Hence, for the atomic pairs passing through the cavity, we can directly express the effective temperature of the cavity in terms of the quantum mutual information using Eq. (6), yielding a square-root dependence, $T_{c a}=T(1-\sqrt{\mathcal{I}(1: 2) \ln 2})$. On the other hand, for low temperatures, classical correlations vanish, as expected, and mutual information, discord and concurrence become identical. In this regime, we find that $\mathcal{I}(1: 2) \simeq \delta(1 \mid 2) \simeq \mathcal{C} \simeq \exp (\beta \hbar(\lambda-\omega))$. It then follows that, for small interaction parameter, $T_{c a}=$ $-T(\beta \hbar \omega)^{-1} \ln \mathcal{I}(1: 2)$.

Thermodynamic efficiency. - We are now in the position to show that quantum correlations can increase

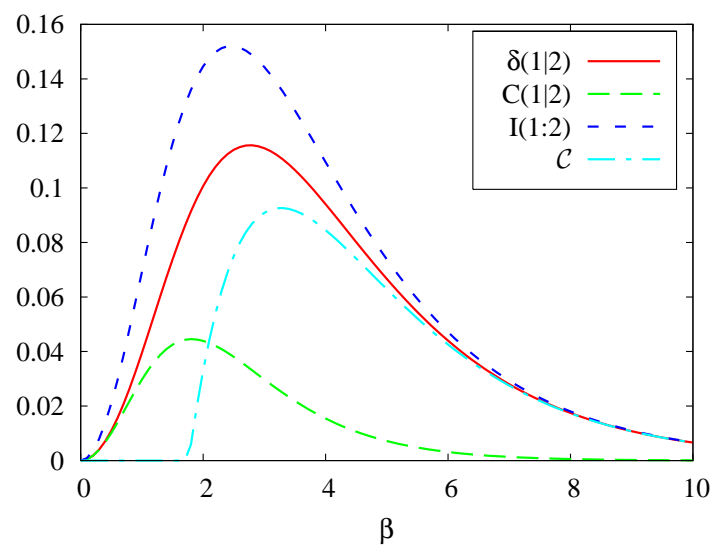

Fig. 4: Four measures of the correlations in the thermal entangled state $\rho$ as a function of the inverse temperature $\beta$ : quantum discord $\delta(1 \mid 2)$, classical correlations $C(1 \mid 2)$, total correlations $\mathcal{I}(1: 2)$ and concurrence $\mathcal{C}$, for $\hbar \omega=1$ and $\hbar \lambda=0.5$. For high temperatures, the concurrence is zero and classical correlations are equal to quantum correlations. By contrast, for low temperatures, classical correlations vanish and $\mathcal{I}(1: 2) \simeq \delta(1 \mid 2) \simeq \mathcal{C}$.

the thermodynamic efficiency above its classical value. We consider a usual Carnot cycle where the cavity is successively put into contact with a high and low temperature reservoir as discussed in detail in Ref. [11]. In the situation where the two atoms of the pairs pass through the cavity, the atomic reservoir is taken to be the cold reservoir, while the opposite choice is made for the one-atom case. For high temperatures, taking into account that $\mathcal{I}(1: 2)=\delta(1 \mid 2)+C(1 \mid 2)$, the thermodynamic efficiency can be written as,

$$
\eta=\eta_{C}+\frac{T_{c}}{T_{h}} \sqrt{(\delta(1 \mid 2)+C(1 \mid 2)) \ln 2} .
$$

The efficiency $\eta$ can thus be decomposed into the classical efficiency $\eta_{C}$ (without correlations), and the contributions from the quantum correlations $\delta(1 \mid 2)$ and the accompanying classical correlations $C(1 \mid 2)$. By contrast, for lower temperatures, since classical correlations vanish and $\mathcal{I}(1: 2) \simeq \delta(1 \mid 2) \simeq \mathcal{C}$, we can directly relate the efficiency to the concurrence of an entangled pair,

$$
\eta=\eta_{C}+\frac{T_{c}}{T_{h}}\left(1+\frac{1}{\beta \hbar \omega} \ln \mathcal{C}\right)
$$

The above equations show that the thermodynamic efficiency in presence of correlations between the two atoms exceeds the classical efficiency $\eta_{C}$ correspondig to uncorrelated atoms. Furthermore, we note that the quantum heat engine can still extract work from the external reservoirs when their temperatures are equal, $T_{c}=T_{h}$ [11]. It should be emphasized that the second law of thermodynamics is not violated even though work can be gained from a single 
reservoir. Indeed the interaction between atoms and cavity field induces a temperature difference between the two only when atomic pairs are correlated, and work has to be provided to prepare the correlated thermal entangled state (2). Finally, it is interesting to mention that a highquality optical cavity with a movable mirror has recently been fabricated with a micromechanical mirror [27].

Conclusion. - We have shown that a beam of quantum correlated two-level atoms can modify detailedbalance between absorption and emission of photons in a single-mode cavity and change its effective temperature. In the limit of small correlations, we have expressed the efficiency the microscopic photo-Carnot engine in terms of the quantum discord and shown that the efficiency exceeds the classical limit given by uncorrelated atoms. Useful work can thus be extracted from quantum correlated, but not necessarily entangled, thermal qubits. These findings show that quantum correlations cannot only be used as a valuable resource in quantum information theory, but also in the realm of quantum thermodynamics.

$$
* * *
$$

This work was supported by the Emmy Noether Program of the DFG (contract LU1382/1-1) and the cluster of excellence Nanosystems Initiative Munich (NIM).

\section{REFERENCES}

[1] M.A. Nielsen and I.L Chuang, Quantum Computation and Quantum Information, (Cambridge University Press, Cambridge, 2000).

[2] C.H. Bennett, D.P. DiVincenzo, C.A. Fuchs, T. Mor, E. Rains, P.W. Shor, J.A. Smolin, and W.K. Wootters, Phys. Rev. A 59 (1999) 1070.

[3] H. Ollivier and W.H. Zurek, Phys. Rev. Lett. 88 (2002) 017901.

[4] M. Horodecki, K. Horodecki, P. Horodecki, R. Horodecki, J. Oppenheim, A. Sen(De), and U. Sen, Phys. Rev. Lett. 90 (2003) 100402.

[5] A. Datta, A. Shaji, and C.M. Caves, Phys. Rev. Lett. 100 (2008) 050502.

[6] B.P. Lanyon, M. Barbieri, M.P. Almeida, and A.G. White, Phys. Rev. Lett. 101, (2008) 200501.

[7] J. Oppenheim, M. Horodecki, P. Horodecki, and R. Horodecki, Phys. Rev. Lett. 89 (2002) 180402.

[8] W.H. Zurek, Phys. Rev. A 67 (2003) 012320.

[9] M. Horodecki, P. Horodecki, R. Horodecki, J. Oppenheim, A. Sen(De), U. Sen, and B.Synak-Radtke, Phys. Rev. A 71 (2005) 062307.

[10] R. Alicki, M Horedecki, P. Horodecki and R. Horodecki, Open Sys. and Information Dyn. 11 (2004) 205.

[11] M.O. Scully, M.S. Zubairy, G.S. Agarwal, and H. Walther, Science 299 (2003) 862

[12] M.A. Nielsen, PhD thesis, Univ. of New Mexico (1998).

[13] M.C. Arnesen, S. Bose, and V. Vedral, Phys. Rev. Lett. 87 (2001) 017901.

[14] G.L. Kamta and A.F. Starace, Phys. Rev. Lett. 88 (2002) 107901.
[15] L. Amico, R. Fazio, A. Osterloh, and V. Vedral, Rev. Mod. Phys. 80 (2008) 517.

[16] S. Ghosh, T.F. Rosenbaum, G. Aeppli, and S.N. Coppersmith, Nature 425 (2003) 48.

[17] T. Vértesi and E. Bene, Phys. Rev. B 73 (2006) 134404.

[18] T.G. Rappoport, L. Ghivelder, J.C. Fernandes, R.B. Guimarães, and M.A. Continentin, Phys. Rev. B 75 (2007) 054422.

[19] S.B. Zheng and G.C. Guo, Phys. Rev. Lett. 85 (2000) 11.

[20] S. Osnaghi, P. Bertet, A. Auffeves, P. Maioli, M. Brune, J.M. Raimond, and S. Haroche, Phys. Rev. Lett. 87 (2001) 037902.

[21] X. Wang, Phys. Rev. A 64 (2001) 012313.

[22] W.P. Schleich, Quantum Optics in Phase Space, (Wiley, New York, 2001).

[23] C. Cohen-Tannoudji, J. Dupont-Roc, and G. Grynberg, Atom-Photon Interactions, (Wiley, New York, 1992).

[24] H.B Callen, Thermodynamics and an Introduction to Thermostatistics (Wiley, New York, 1985).

[25] L. Henderson, V. Vedral, J. Phys. A 34 (2001) 6899.

[26] S. Hamieh, R. Kobes, H. Zaraket, Phys. Rev. A 70 (2004) 052325.

[27] H.R. Böhm, S. Gigan, G. Langer, J.B. Hertzberg, F. Blaser, D. Bäuerle, K.C. Schwab, A. Zeilinger, and M. Aspelmeyer, Appl. Phys. Lett. 89 (2006) 223101. 